\title{
Excitation and emission spectra of in silico red-shift predicted iLOV mutants
}

\author{
Pierre Wehler ${ }^{1,2}$ and Mehmet Ali Öztürk ${ }^{1,2, *}$
}

1 Institute of Biology II, University of Freiburg, Schänzlestraße 1, 79104 Freiburg, Germany

2 Centers for Biological Signalling Studies BIOSS and CIBSS, University of Freiburg, Schänzlestraße 1, 79104 Freiburg, Germany

* Corresponding author: Mehmet Ali Öztürk, Institute of Biology II University of Freiburg, Schänzlestraße 1, 79104 Freiburg, +49 761203 2787, mehmet.oeztuerk@bioss.unifreiburg.de

\begin{abstract}
iLOV is a flavin mononucleotide (FMN)-binding fluorescent protein (FbFP) used for in vivo cellular imaging similar to the green fluorescent protein (GFP). Contrary to GFP, iLOV fluoresces independently of molecular oxygen, making its usage in low-oxygen conditions possible. Moreover, iLOV is smaller than GFP, increasing the likelihood of retaining full functionality when creating fusions with proteins of interest. Nonetheless, GFP remains to date the most widely used FP in molecular biology, also due to the availability of multiple spectrally tuned variants allowing multi-color imaging experiments. To expand the range of applications of iLOV, spectrally tuned red-shift variants are desirable to have reduced phototoxicity and better tissue penetration. In this short report, we experimentally tested two iLOV mutants, iLOV ${ }^{\mathrm{L} 470 \mathrm{~T} / \mathrm{Q} 489 \mathrm{~K}}$ and $\mathrm{iLOV}^{\mathrm{V} 392 \mathrm{~K} / \mathrm{F} 410 \mathrm{~V} / \mathrm{A} 426 \mathrm{~S}}$, which were previously computationally proposed by Khrenova et al. (DOI: 10.1021/acs.jpcb.7b07533) to have redshifted excitation and emission spectra. We found that only the triple mutant has moderately red-shifted excitation and emission spectra. Both mutants exhibit strongly decreased in vivo fluorescence intensity, which impedes their employment in live cell imaging. Finally, we show that the single $\mathrm{iLOV}^{\mathrm{V} 392 \mathrm{~K}}$ mutant suffices to red-shift the emission spectrum.
\end{abstract}




\section{Introduction}

Fluorescent proteins (FPs) have revolutionized cell biology by enabling researchers to investigate dynamic cellular processes in real time ${ }^{1}$. The most widely used FP is GFP and its spectrally shifted and/or engineered variants ${ }^{1}$, for instance further optimized to more rapidly mature $(\mathrm{sfGFP})^{2}$ or more brightly fluoresce (mNeonGreen $)^{3}$. Despite their usefulness, FPs of the GFP family have some limitations: (a) they depend on molecular oxygen for chromophore formation, which impedes their use to visualize processes under hypoxic or anoxic conditions ${ }^{4-6}$; (b) are relatively large $(\sim 25 \mathrm{kDa})$, which can be problematic in some applications ${ }^{7,8}$, (c) and are mostly sensitive to $\mathrm{pH}^{9}$. While $\mathrm{pH}$-resistant GFP variants have been engineered ${ }^{9}$, oxygen-dependence and size are mostly unchangeable features. FMNbinding fluorescent proteins (FbFPs) overcome these limitations and thus are valuable alternatives to GFP and its variants ${ }^{10,11}$. FbFPs consist of a small light oxygen voltage (LOV) domain $(\sim 11 \mathrm{kDa})$ modified to emit green fluorescence when light in the UV-A-blue range reaches the non-covalently bound FMN chromophore ${ }^{12}$.

One prominent FbFP is iLOV, a derivative of the LOV2 domain of Arabidopsis thaliana ${ }^{7}$. Several rounds of DNA shuffling led to a mutation in a key cysteine residue and triggering fluorescence emission ${ }^{7}$. While FbFPs have been used in molecular imaging for more than a decade $^{13}$, they suffer from autofluorescence and relatively weak fluorescent intensity issues which have not been improved until very recently ${ }^{14,15}$. Engineering a red-shifted iLOV could address autofluorescence problems by shifting the excitation and emission peaks away from the cellular autofluorescence. Additionally, potential phototoxicity due to prolonged bluelight application could be minimized, and applications which require tissue penetration could be enabled. Moreover, a red-shifted FbFP variant could be used orthogonally to other FbFPs and would potentially allow for multicolor imaging and FRET-based biosensors.

By using the observation of the presence of positively charged residues in close proximity of the chromophore, which is a motif in red-light fluorescent proteins like Rtms $5^{16}$ and mKeima $^{17}$, Khrenova et al. previously applied quantum mechanics / molecular mechanics $(\mathrm{QM} / \mathrm{MM})$ simulations and proposed that $\mathrm{iLOV}^{\mathrm{Q} 489 \mathrm{~K}}$ mutant could represent $\sim 50 \mathrm{~nm}$ red shift of excitation and emission band maxima of $\mathrm{iLOV}^{18}$. While their rationale was based on the design of positively charged amino group of K489 next to chromophore stabilizing the $\pi$ electron system of the FMN in the excited state, later Davari et al. computationally and experimentally showed that K489 is mostly populated in an open conformer which is far from 
the chromophore and in fact $\mathrm{iLOV}^{\mathrm{Q} 489 \mathrm{~K}}$ is blue shifted ${ }^{19}$. In a follow up study, Khrenova et al. ${ }^{20}$ applied a second round of QM/MM calculations to find iLOV mutants that have more stabilized lysine residues next to the chromophore than previous $\mathrm{iLOV}^{\mathrm{Q} 489 \mathrm{~K}}$ mutant and they proposed that $\mathrm{iLOV}^{\mathrm{L} 470 \mathrm{~T} / \mathrm{Q} 489 \mathrm{~K}}$ and $\mathrm{iLOV}^{\mathrm{V} 392 \mathrm{~K} / \mathrm{F} 410 \mathrm{~V} / \mathrm{A} 426 \mathrm{~S}}$ mutants could result in $\sim 50 \mathrm{~mm}$ red shift for both excitation - emission spectra of iLOV. A shift by this size would open up new potential applications for iLOV and FbFPs in general. In this short report, we experimentally tested further QM/MM calculations of Khrenova et al. ${ }^{20}$ by measuring the excitation and emission spectra of purified iLOV variants $\left(\mathrm{WT}, \mathrm{iLOV}^{\mathrm{L} 470 \mathrm{~T} / \mathrm{Q} 489 \mathrm{~K}}\right.$ and $\mathrm{iLOV}^{\mathrm{V} 392 \mathrm{~K} / \mathrm{F} 410 \mathrm{~V} / \mathrm{A} 426 \mathrm{~S}}$ and $\left.\mathrm{iLOV}^{\mathrm{V} 392 \mathrm{~K}}\right)$.

\section{Material and Methods}

\section{Plasmid construction}

Constructs were generated using classical restriction enzyme cloning or golden gate cloning. Constructs and oligonucleotide sequences used in this study are listed in Supplementary Tables I and II, respectively.

The ilov gene was amplified with primers OL01 and OL02 from iLOV-N1, gift from Michael Davidson (Addgene plasmid \#54673; http://n2t.net/addgene:54673; RRID:Addgene_54673) and cloned into pcDNA3.1(+) using HindIII and EcoRI restriction sites, yielding plasmid PL01. PL01 was used as a template to clone all iLOV variants used in this study. Single-point mutations Q489K and V392K were created via overhang PCR using primer pairs OL01OL03 and OL02-OL04 yielding PL02 and PL04, respectively. The L470T mutation was introduced into PL02 using golden gate cloning. The first fragment was amplified with primers OL01 and OL10, the second fragment with primers OL09 and OL03. Amplicons were digested with HindIII and BpiI, or BpiI and EcoRI, respectively, and cloned into pcDNA3.1(+) yielding PL03. The A426S mutation was inserted into PL04 by addition of a $\mathrm{XbaI}$ restriction site into the coding sequence. The first part was amplified using primers OL04 and OL06, the second part was amplified using primers OL05 and OL02 and cloned into pcDNA3.1(+) yielding PL05. The F410V mutation was introduced using golden gate cloning. The first fragment was amplified with primers OL04 and OL08, the second fragment with primers OL02 and OL07. Amplicons were digested with HindIII and BpiI or BpiI and EcoRI, respectively, and cloned into pcDNA3.1(+) yielding PL06.

For expression in and purification from Escherichia coli, the ilov, ilov $v^{L 470 T / Q 489 K}$, ilov $v^{V 392 K}$ and 
ilov $v^{V 392 K / F 410 V / A 426 S}$ genes were amplified using primers OL11 or OL12 (for mutants containing the V392K mutation) and OL13, and then cloned into pET28a using NdeI and XhoI restriction sites, yielding PL07-PL10. The sequence of all constructs was verified using Sanger sequencing.

\section{Protein expression and purification}

Each pET28a plasmid harbouring one iLOV construct was transformed into E. coli Rosetta $^{\mathrm{TM}}$ (DE3) pLysS cells for protein expression. A large expression culture (1L LB containing 0.1 $\mathrm{mg} / \mathrm{mL}$ kanamycin) was started with an optical density $\left(\mathrm{OD}_{600}\right)$ of 0.05 from an overnight culture and was grown at $37^{\circ} \mathrm{C}$ at $180 \mathrm{rpm}$ until it reached an $\mathrm{OD}_{600}$ of 0.8 . IPTG (Isopropyl- $\beta-D$-thiogalactopyranosid) was then added to the culture to a final concentration of $1 \mathrm{mM}$, and the culture was further grown at $18{ }^{\circ} \mathrm{C}$ overnight. The next morning, cells were harvested via centrifugation at $5000 \times \mathrm{g}$ for 30 minutes. The pellets were resuspended in lysis buffer $(50 \mathrm{mM}$ potassium phosphate $\mathrm{pH} 8.0,300 \mathrm{mM} \mathrm{NaCl}$ and $10 \mathrm{mM}$ imidazole $\mathrm{pH}$ 8.0) containing one tablet of cOmplete ${ }^{\mathrm{TM}}$ protease inhibitor cocktail (Roche), and lysed by sonication. The lysate was clarified by centrifugation at 20,000 rpm at $4{ }^{\circ} \mathrm{C}$ and loaded onto an IMAC nickel column $(1 \mathrm{~mL})$ using the Bio-Rad NGC automated liquid chromatography system. The column was washed with wash buffer (same as lysis buffer but with $20 \mathrm{mM}$ imidazole and $10 \%$ glycerol) and eluted with elution buffer (same as lysis buffer but the $\mathrm{pH}$ of potassium phosphate is 7.5 with further addition of $10 \%$ glycerol and $500 \mathrm{mM}$ imidazole). Finally, the elution buffer was replaced with a storage buffer (50 mM HEPES-KOH pH 7.25, $150 \mathrm{mM} \mathrm{KCl,} 10 \%$ glycerol and 0.1 mM EDTA pH8.0) using a P-6 desalting column (10 mL). The purified protein was aliquoted and the aliquots were stored at $-80{ }^{\circ} \mathrm{C}$.

\section{Spectroscopy}

Excitation and emission spectra were measured with a fluorescence spectrophotometer (Tecan Infinite M200). $200 \mu \mathrm{L}$ of each purified protein (final concentration $=333 \mu \mathrm{g} / \mathrm{mL}$ ) were dispensed into a well of a black 96-well plate. $200 \mu \mathrm{L}$ of the storage buffer was used as blank. For the excitation spectrum, excitation was carried out from $420 \mathrm{~nm}$ to $490 \mathrm{~nm}$ and fluorescence was measured at $530 \mathrm{~nm}$ (step size $1 \mathrm{~nm}$ ). For the emission spectrum, excitation 
was fixed at $450 \mathrm{~nm}$, and the fluorescent signal was measured from $480 \mathrm{~nm}$ to $570 \mathrm{~nm}$ (step size $2 \mathrm{~nm})$.

\section{Cell culture and transient transfection}

Human non-small cell lung carcinoma cells (H1299; kindly provided by Alexander Loewer, Max Delbrück Center for Molecular Medicine, Berlin) were maintained in RPMI 1640 media supplemented with $10 \%$ foetal bovine serum, $100 \mathrm{U} / \mathrm{ml}$ penicillin and $100 \mu \mathrm{g} / \mathrm{ml}$ streptomycin. Cells were cultivated at $37{ }^{\circ} \mathrm{C}$ and $5 \% \mathrm{CO}_{2}$ and were passaged when reaching $\sim 90 \%$ confluence.

For microscopy experiments, H1299 cells were seeded in $\mu$-Slide 8-well chambers (Ibidi $\mathrm{GmbH}$ ) at densities of $\sim 15000$ per well. The following day, the samples were transfected with $150 \mathrm{ng}$ of construct DNA using Lipofectamin 2000 (ThermoFisher Scientific) according to the manufacturer's manual. Samples were imaged 36 hours post-transfection.

\section{Fluorescence microscopy}

Cells were imaged at $37{ }^{\circ} \mathrm{C}$ and $5 \% \mathrm{CO}_{2}$ in $\mu$-Slide 8 well chambered coverslips (Ibidi $\mathrm{GmbH}$ ) on a Zeiss AxioObserver wide-field microscope equipped with an incubation chamber, a 40x air objective (0.7 NA), the Colibri.2 LED light source and a filter set for eGFP.

\section{Data processing}

Analysis of spectral data was carried out using Python ${ }^{21}$, particularly the pandas library ${ }^{22}$. The blank was subtracted from the respective values and the respective spectra were then normalized to their highest value. Plots were generated using the matplotlib library ${ }^{23}$.

\section{Results}

Emission spectrum of purified $i L O V^{L 470 T / Q 489 K}$ and $i L O V^{V 392 K / F 410 V / 4426 S}$ mutants show minimal red shift

In order to verify the computationally predicted shift in the excitation and emission spectra of the two proposed iLOV mutants (Figure 1), we cloned WT ilOV, iLOV ${ }^{\mathrm{L} 470 \mathrm{~T} / \mathrm{Q} 489 \mathrm{~K}}$ and $\mathrm{iLOV}^{\mathrm{V} 392 \mathrm{~K} / \mathrm{F} 410 \mathrm{~V} / \mathrm{A} 426 \mathrm{~S}}$ into a bacterial expression plasmid carrying an N-terminal 6xHis-tag and purified the iLOV variants via Nickel affinity chromatography. SDS gel of the purified proteins indicate that iLOV mutants may cause multimers and/or aggregates (Supplementary Figure

$1)$. 
In contrast to the computations of Khrenova et al. ${ }^{20}$, our experiments indicated that the excitation peak of $\mathrm{iLOV}^{\mathrm{L} 470 \mathrm{~T} / \mathrm{Q} 489 \mathrm{~K}}$ was in fact slightly blue shifted with the maximum of 443 $\mathrm{nm}$ (WT $448 \mathrm{~nm}$ ) (Figure 2 and Table 1). The emission peak was red shifted by $6 \mathrm{~nm}$ to 506 $\mathrm{nm}(\mathrm{WT} 500 \mathrm{~nm})$. The second mutant, iLOV ${ }^{\mathrm{V} 392 \mathrm{~K} / \mathrm{F} 410 \mathrm{~V} / \mathrm{A} 426 \mathrm{~S}}$, was red-shifted in both excitation $(455 \mathrm{~nm})$ and emission $(514 \mathrm{~nm})$ peaks. However, the shift of the excitation maxima was 14 $\mathrm{nm}$ (from $500 \mathrm{~nm}$ to $514 \mathrm{~nm}$ ), which was lower compared to the prediction of $35 \mathrm{~nm}$ from Khrenova et al. ${ }^{20}$. As a result, among computationally proposed mutants, only triple mutant showed a slight excitation and emission red-shift (Table 1). While it is not normalized with the flavin load of each mutant, experiments conducted with purified proteins in the same conditions indicate orders of magnitude reduced fluorescence intensity of the mutants compared to WT iLOV (Supplementary Figure 2).

$i L O V^{V 392 K}$ has similar excitation and emission properties as iLOV V392K/F410V/426S

Of the two characterized mutants, $\mathrm{iLOV}^{\mathrm{V} 392 \mathrm{~K} / \mathrm{F} 410 \mathrm{~V} / \mathrm{A} 426 \mathrm{~S}}$ exhibited more favourable red-shift spectral properties. To evaluate the contribution of an individual mutation to the spectral shift and the reduced brightness, we investigate $\mathrm{iLOV}^{\mathrm{V} 392 \mathrm{~K}}$ single mutant, which has neither computationally nor experimentally characterized before.

The excitation - emission spectral properties of $\mathrm{iLOV}^{\mathrm{V} 392 \mathrm{~K}}$ and $\mathrm{iLOV}^{\mathrm{V} 392 \mathrm{~K} / \mathrm{F} 410 \mathrm{~V} / \mathrm{A} 426 \mathrm{~S}}$ were similar (Figure 3, Table 1) and $\mathrm{iLOV}^{\mathrm{V} 392 \mathrm{~K}}$ had higher fluorescence intensity in the same experimental conditions in which flavin load was not normalized (Supplementary Figure 3). Apparently, mutation of V392K was sufficient to cause the red-shift in excitation peak (456 $\mathrm{nm})$ to the same extent as $\mathrm{iLOV}^{\mathrm{V} 392 \mathrm{~K} / \mathrm{F} 410 \mathrm{~V} / \mathrm{A} 426 \mathrm{~S}}(455 \mathrm{~nm})$, and a mildly stronger shift in the emission maxima (518 nm vs $514 \mathrm{~nm}$ ). As iLOV ${ }^{\mathrm{V} 392 \mathrm{~K}}$ was sufficient for total red light shift of the triple mutant, we did not analyze other mutants. We further evaluated whether any of the mutants $\left(\mathrm{iLOV}^{\mathrm{V} 392 \mathrm{~K}}\right.$, $\mathrm{iLOV}^{\mathrm{L} 470 \mathrm{~T} / \mathrm{Q} 489 \mathrm{~K}}$ or $\left.\mathrm{iLOV}^{\mathrm{V} 392 \mathrm{~K} / \mathrm{F} 410 \mathrm{~V} / \mathrm{A} 426 \mathrm{~S}}\right)$ is sufficient for in vivo fluorescence experiments and we decided to test WT iLOV and these variants in mammalian cells.

\section{In vivo fluorescence analysis of $i L O V$ variants}

We selected the non-small lung cancer cell line H1299 for evaluating the in vivo fluorescence signal of iLOV mutants. We were able to get a WT iLOV fluorescence signal with $150 \mathrm{ng}$ DNA transfection and 36 hours of expression. However none of the iLOV mutants $\left(\mathrm{iLOV}^{\mathrm{V} 392 \mathrm{~K}}, \mathrm{iLOV}^{\mathrm{L} 470 \mathrm{~T} / \mathrm{Q} 489 \mathrm{~K}}\right.$ or $\mathrm{iLOV}^{\mathrm{V} 392 \mathrm{~K} / \mathrm{F} 410 \mathrm{~V} / \mathrm{A} 426 \mathrm{~S}}$ ) exhibited any fluorescence signal in these conditions, indicating that while their excitation and emission can shift to red, their 
fluorescence intensity is significantly reduced (Figure 4).

\section{Discussion}

In this study, we set out to analyze the excitation and emission spectra of the two iLOV mutants $\mathrm{iLOV}^{\mathrm{L} 470 \mathrm{~T} / \mathrm{Q} 489 \mathrm{~K}}$ and $\mathrm{iLOV}^{\mathrm{V} 392 \mathrm{~K} / \mathrm{F} 410 \mathrm{~V} / \mathrm{A} 426 \mathrm{~S}}$, which were computationally predicted to be red-shifted up to $50 \mathrm{~nm}^{20}$. However, our in vitro validation could not support the predicted extent of the shifts. Initially, Khrenova et al. predicted the single mutant iLOV ${ }^{\mathrm{Q} 489 \mathrm{~K}}$ to be shifted in excitation and emission peak by $52 \mathrm{~nm}$ and $97 \mathrm{~nm}$, yet the measured excitation and emission peaks exhibited a blue-shift of about $10 \mathrm{~nm}^{18,19}$, highlighting the disagreement between prediction and experimental validation. Even though the QM/MM modelling

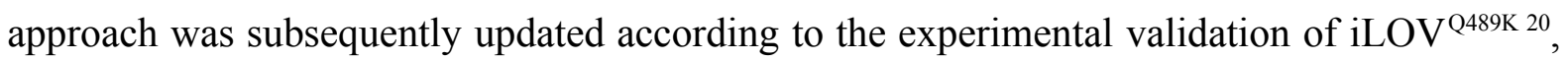
it seems not able to capture the complexity of the interaction of ILOV and its chromophore completely, given the discrepancy between prediction and the experimental validation for the two mutants characterized here. From a computational perspective, iLOV may have higher time scale structural rearrangements which require much longer simulation trajectories to capture the effects of the applied mutans.

The mechanistic understanding of the spectral effects of the tested iLOV mutants requires more detailed molecular characterization efforts like recently applied by Goncharov et al. ${ }^{24}$, Remeeva et al. ${ }^{25}$ and Röllen et al. ${ }^{26}$. In the latter, authors have shown that $\mathrm{iLOV}^{\mathrm{V} 392 \mathrm{~T} / \mathrm{Q} 489 \mathrm{~K}}$ has a slight red shift with emission spectrum maxima of $502 \mathrm{~nm}$. Its corresponding mutant $\mathrm{CagFbFP}^{\mathrm{Q} 148 \mathrm{~K} / 152 \mathrm{~T}}$ (emission maximum $504 \mathrm{~nm}$ ) and another blue shifted variant $\mathrm{CagFbFP}^{\mathrm{Q} 148 \mathrm{~K}}$ (emission maximum $491 \mathrm{~nm}$ ) were used in fluorescence microscopy and successfully separated from each other. Less efficient protein folding, multimerization and lower FMN affinity to iLOV could be potential mechanisms of the discrepancy of the experimental and computational data reported here, which remains to be tested. In this study, we could identify the single mutant iLOV ${ }^{\mathrm{V} 392 \mathrm{~K}}$, to our knowledge the FbFP displaying the most red-shifted emission peak $(518 \mathrm{~nm})$. This mutant displays similar excitation and emission characteristics as $\mathrm{LLOV}^{\mathrm{V} 392 \mathrm{~K} / \mathrm{F} 410 \mathrm{~V} / \mathrm{A} 426 \mathrm{~S}}$.

Previous efforts to improve the usability of iLOV resulted in the generation of phiLOV, an iLOV derivative which possesses a superior photostability, thus tackling one of the major drawbacks in using $\mathrm{iLOV}^{27}$. Recently improved brightness of iLOV variants have been reported $^{14,15}$. It remains to be seen whether the introduced mutations responsible for the increased photostability and brightness of new iLOV variants can be applied to improve the 
visualization of $\mathrm{iLOV}^{\mathrm{V} 392 \mathrm{~K}}$, without interfering with its excitation and emission spectra.

\section{Declaration of conflict of interest}

The authors have no conflict of interest to declare.

\section{Acknowledgments}

We thank Alexander Löwer (Max Dellbrück Center for Molecular Medicine, Berlin) for sharing the H1299 cell line, and Michael Davidson for sharing his iLOV-N1 vector with us.

\section{Figures and legends}
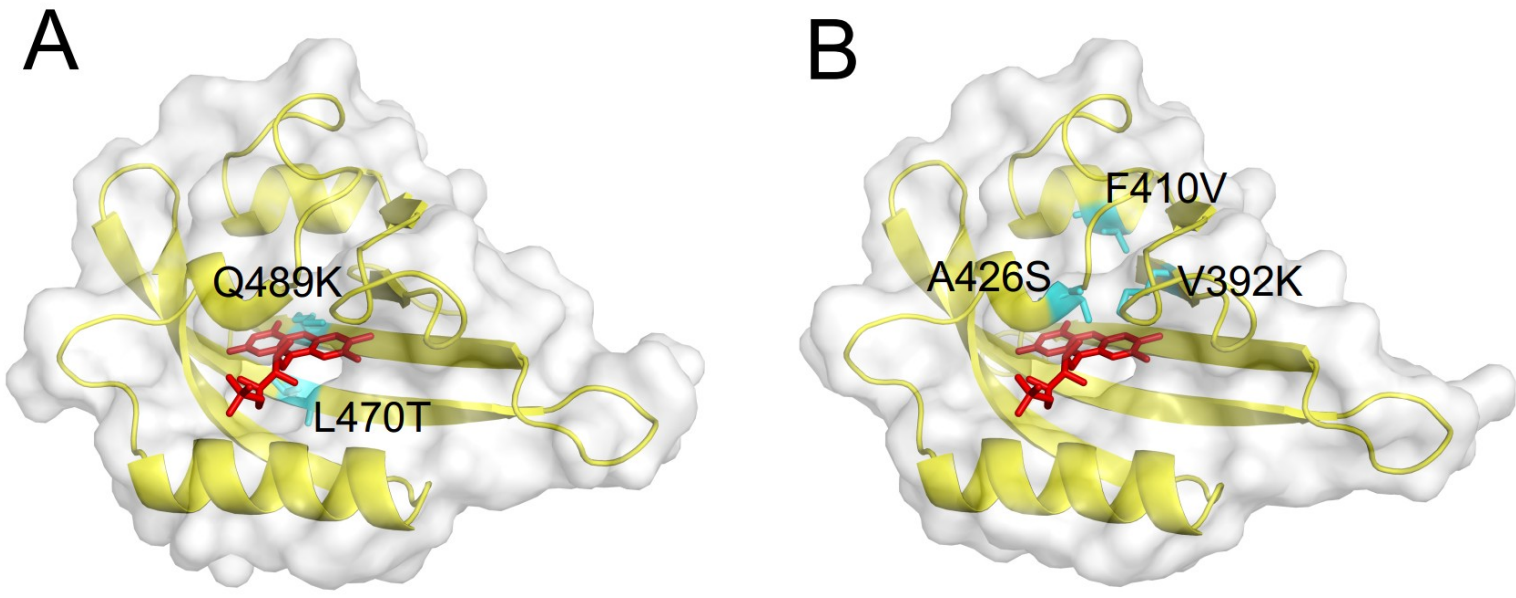

Figure 1: Model structures of iLOV ${ }^{\mathrm{L} 470 \mathrm{~T} / \mathrm{Q} 489 \mathrm{~K}}$ and $\mathrm{iLOV}^{\mathrm{V392K/F410V/A426S}}$ mutants. Cartoon and surface representation of iLOV structures with applied mutations (cyan). Mutant iLOV model structures (yellow) were generated by Swiss-Model web-server ${ }^{28}$ and FMN structures (red) were placed by aligning model outputs to WT iLOV X-ray structure (PDB id: 4EEP). Figures were generated by PyMOL ${ }^{29}$. 

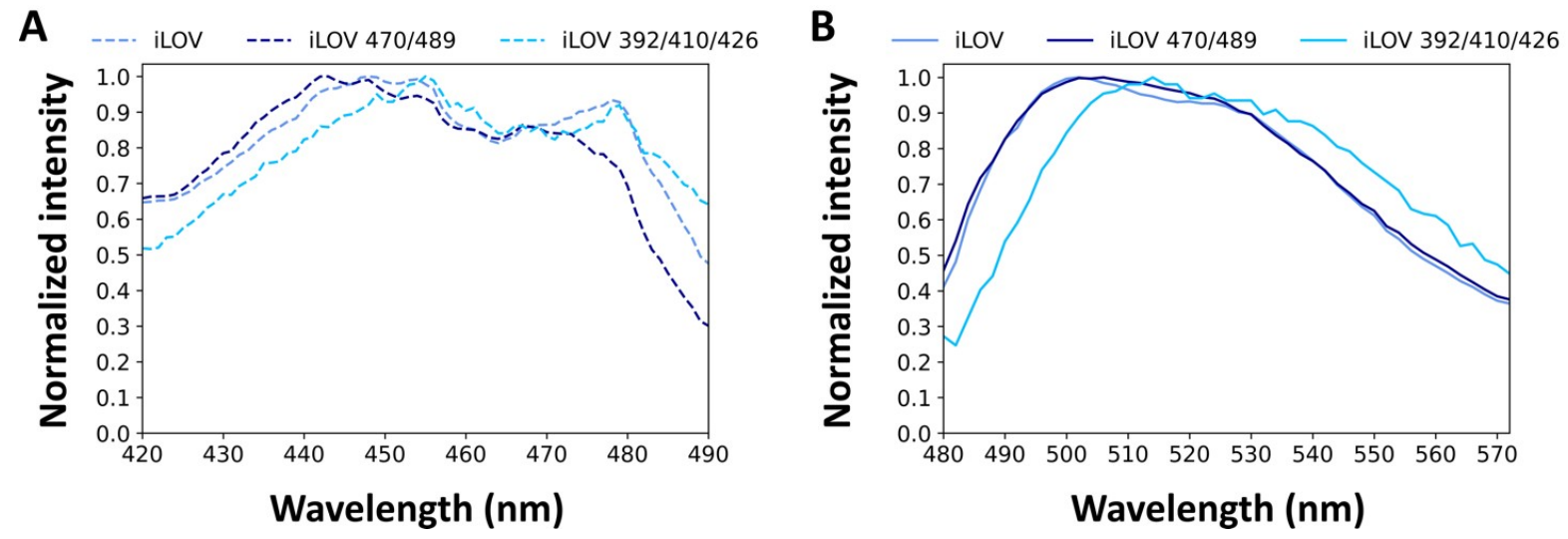

Figure 2: Excitation and emission spectra of purified iLOV variants. Excitation (A) and emission (B) spectra of the indicated iLOV variants. Values of the respective spectra were corrected by subtracting the blank (storage buffer), and then normalized to the highest value of the respective spectra.
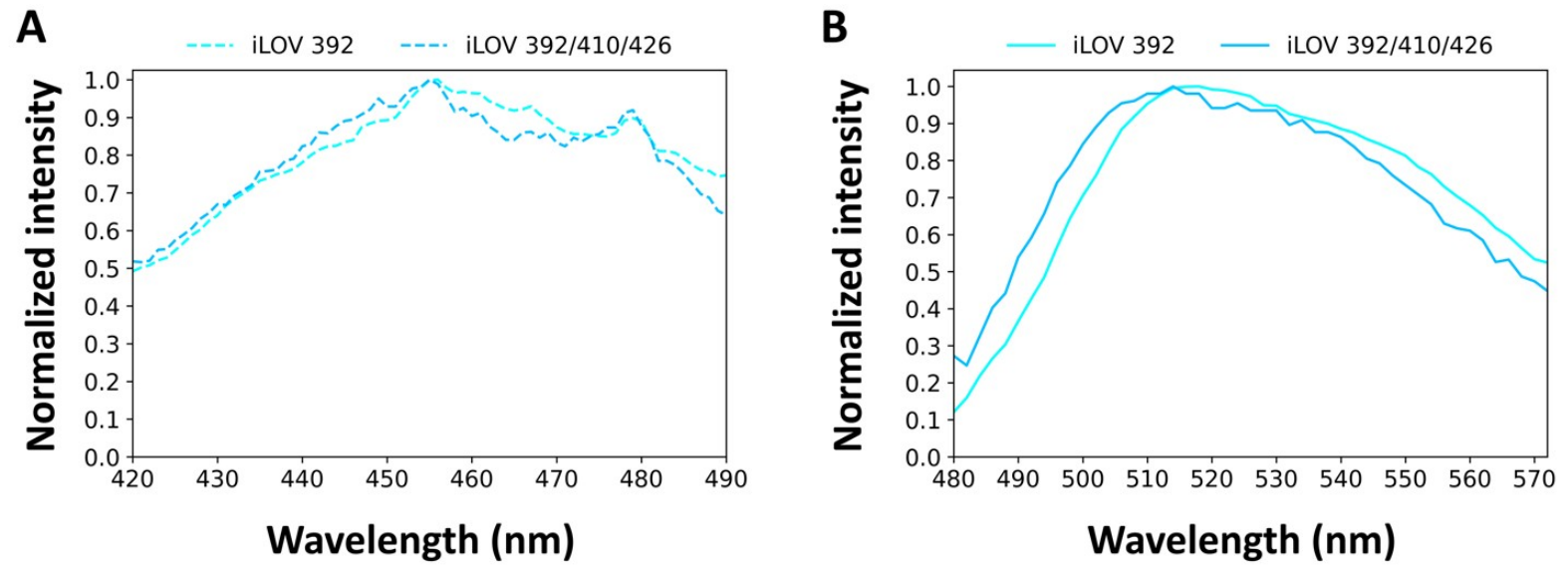

Figure 3: Comparison of the excitation - emission spectra of $\operatorname{iLOV}^{\mathrm{V} 392 \mathrm{~K}}$ and iLOV ${ }^{\mathrm{V} 392 \mathrm{~K} / \mathrm{F410V/A426S}}$ mutants. Excitation (A) and emission (B) spectra of the indicated iLOV variants. Values of the respective spectra were corrected by subtracting the blank (storage buffer), and then normalized to the highest value of the respective spectrum $(A, B)$. 


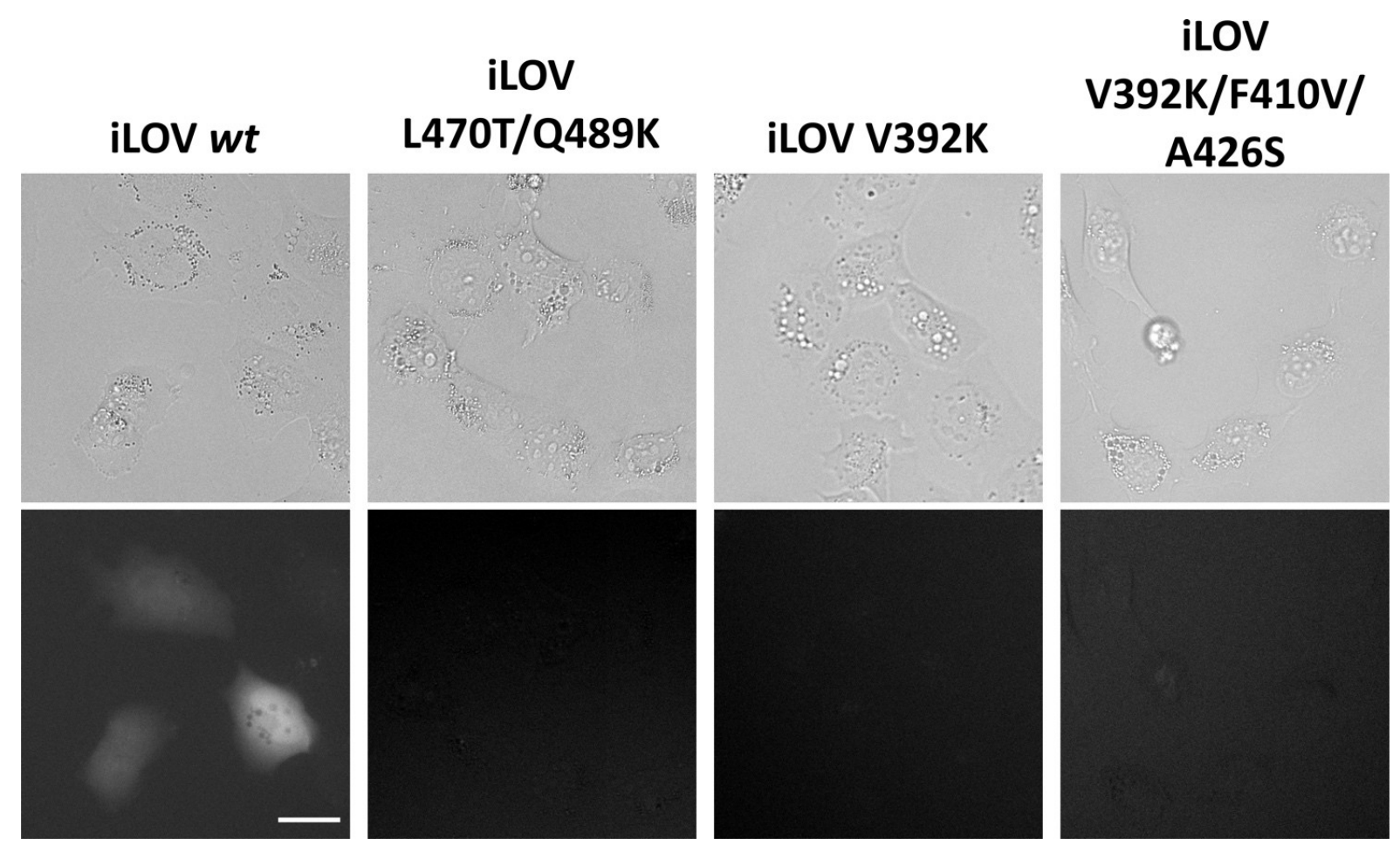

Figure 4: Analysis of iLOV variants in fluorescence microscopy. Representative bright field (top) and eGFP (bottom) images of H1299 cells transiently transfected with the indicated iLOV construct. Scale bar for all micrographs is $30 \mu \mathrm{m}$.

Table 1: Summary of the measured excitation and emission peaks of iLOV variants. Overview of excitation and emission peaks of iLOV variants measured in this study and previously predicted by Khrenova et al. ${ }^{20}$.

\begin{tabular}{ccccc}
\hline iLOV & WT & L470T/Q489K & V392K/F410V/A426S & V392K \\
\hline $\begin{array}{c}\text { Xmax excitation } \\
(\mathbf{n m})\end{array}$ & 448 & 443 (predicted: 475$)$ & 455 (predicted: 498$)$ & 456 \\
$\begin{array}{c}\text { Xmax emission } \\
(\mathbf{n m})\end{array}$ & 500 & 506 (predicted: 539$)$ & 514 (predicted 535) & 518 \\
& & &
\end{tabular}




\section{Supplementary information}

Amino acid sequences of the iLOV variants used in this study are given below:

\section{WT iLOV}

FIEKNFVITDPRLPDNPIIFASDGFLELTEYSREEILGRNARFLQGPETDQATVQKIRDAI RDQRETTVQLINYTKSGKKFWNLLHLQPVRDQKGELQYFIGVQLDGSDHV

\section{iLOV $^{\mathrm{L} 470 \mathrm{~T} / \mathrm{Q} 489 \mathrm{~K}}$}

FIEKNFVITDPRLPDNPIIFASDGFLELTEYSREEILGRNARFLQGPETDQATVQKIRDAI RDQRETTVQLINYTKSGKKFWNLTHLQPVRDQKGELQYFIGVKLDGSDHV

iLOV $\mathrm{V392K/F410V/A426S}$

FIEKNFKITDPRLPDNPIIFASDGVLELTEYSREEILGRNSRFLQGPETDQATVQKIRDA IRDQRETTVQLINYTKSGKKFWNLLHLQPVRDQKGELQYFIGVQLDGSDHV

\section{iLOV $^{\mathrm{V} 392 \mathrm{~K}}$}

FIEKNFKITDPRLPDNPIIFASDGFLELTEYSREEILGRNARFLQGPETDQATVQKIRDA IRDQRETTVQLINYTKSGKKFWNLLHLQPVRDQKGELQYFIGVQLDGSDHV 


\section{$80 \mathrm{kD}$}

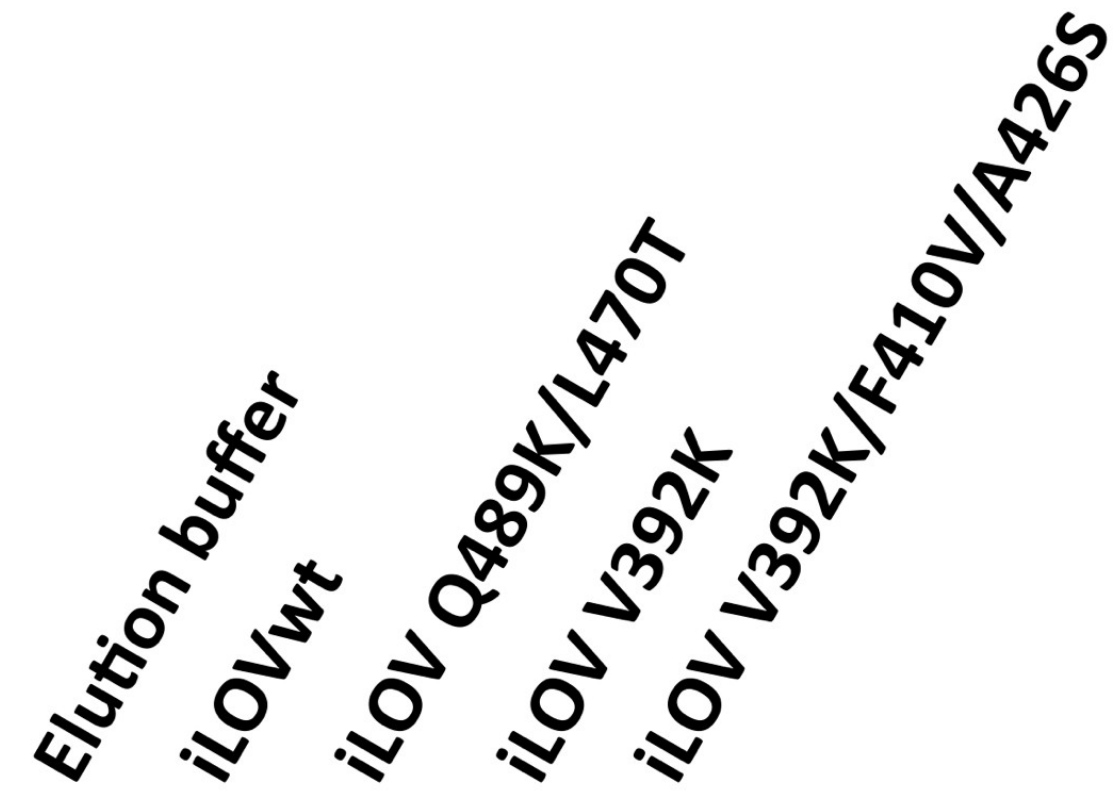

\section{$10 \mathrm{kD}$}

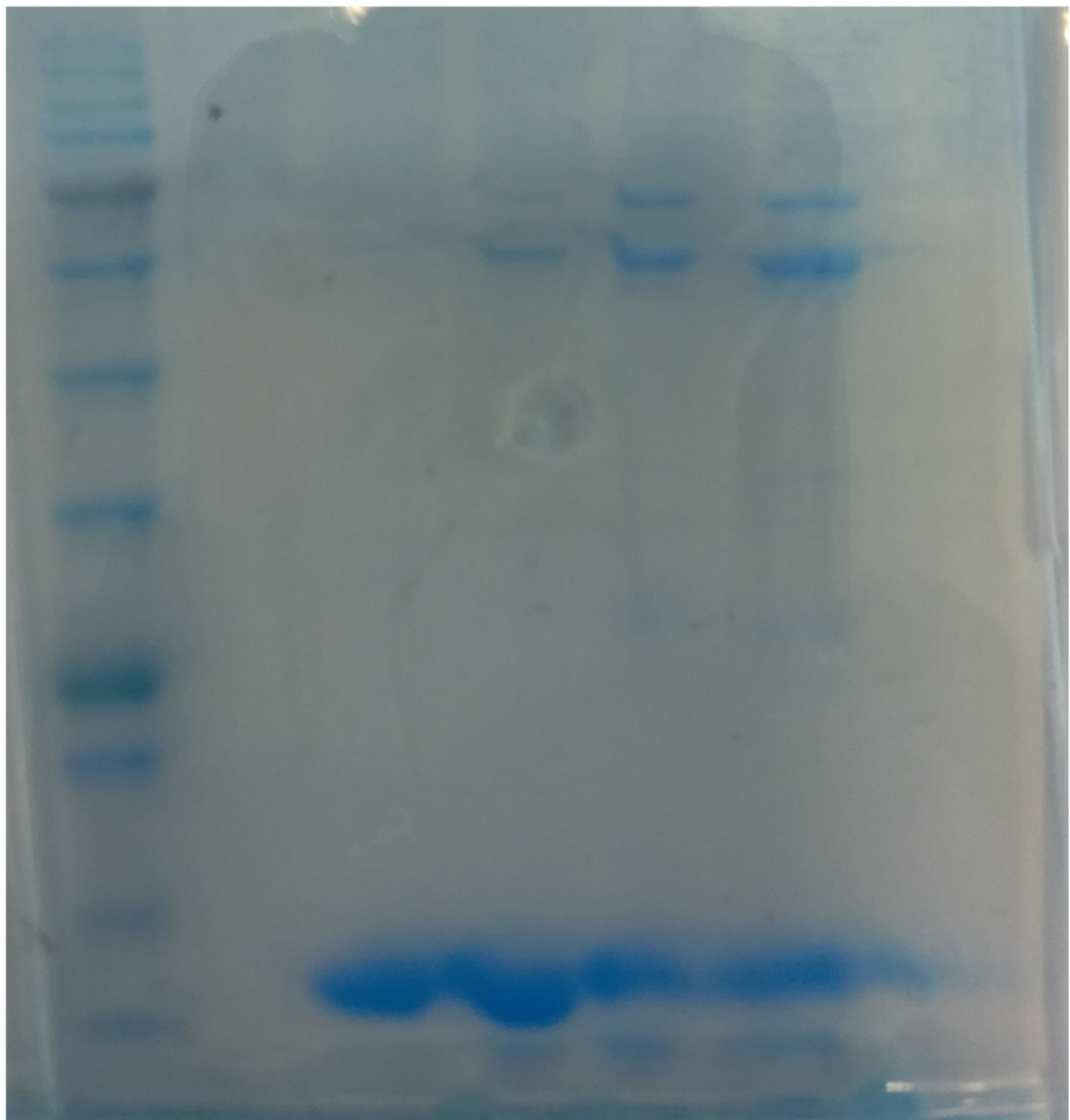

Supplementary Figure 1: SDS-Gel of purified proteins. $1 \mu \mathrm{g}$ of the indicated purified protein was diluted in a $10 \mu 1$ storage buffer, boiled and run on a SDS gel. 

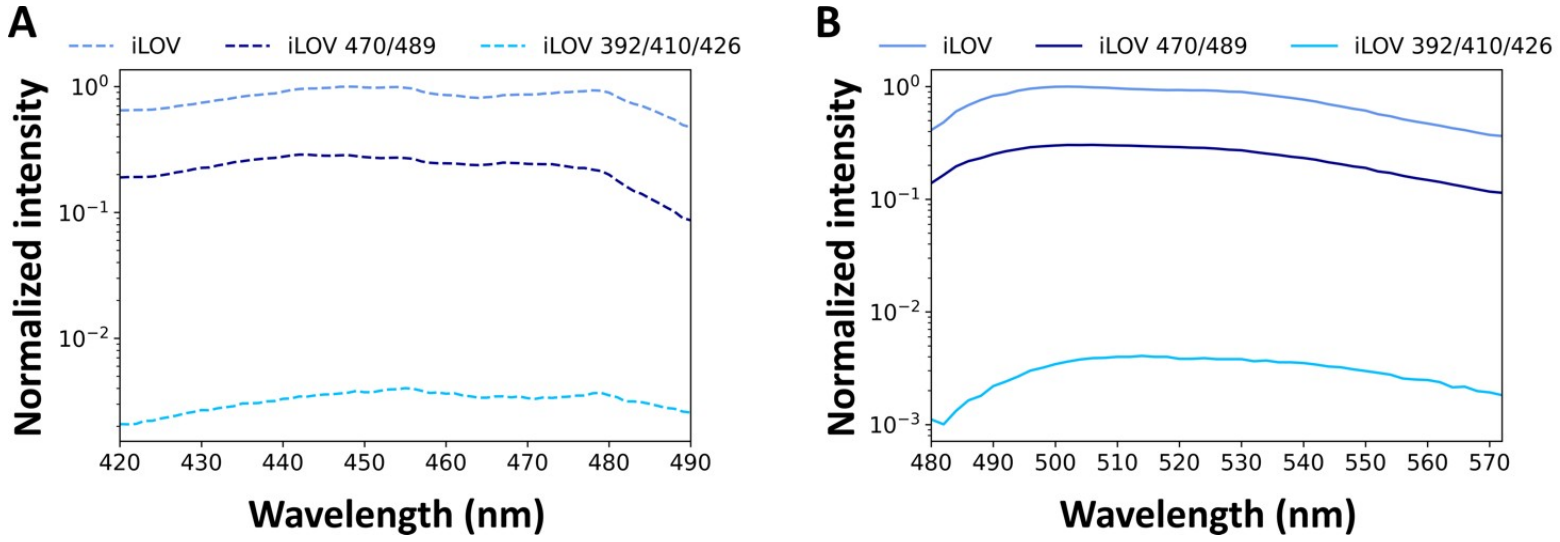

Supplementary Figure 2: Fluorescence intensities of iLOV variants Excitation (A) and emission (B) spectra of the indicated iLOV variants. Values of the respective spectra were corrected by subtracting the blank (storage buffer), and then normalized to the highest value of all three spectra, here iLOV.
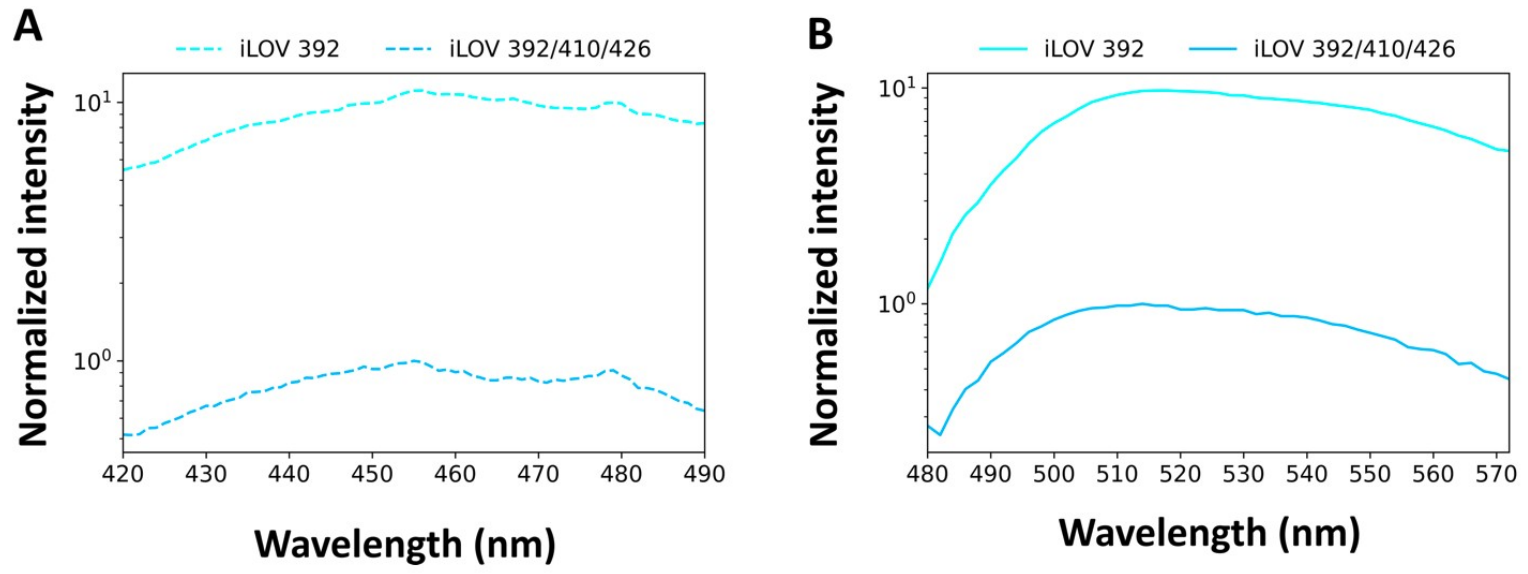

\section{Supplementary Figure 3: Fluorescence intensities of iLOV ${ }^{\mathrm{V} 392 \mathrm{~K}}$ and $\operatorname{iLOV}^{\mathrm{V} 392 \mathrm{~K} / \mathrm{F} 410 \mathrm{~V} / \mathrm{A} 426 \mathrm{~S}}$}

Excitation (A) and emission (B) spectra of the indicated iLOV variants. Values of the respective spectra were corrected by subtracting the blank (storage buffer), and then normalized to the highest value of the respective spectrum of $\mathrm{ILOV}^{\mathrm{V} 392 \mathrm{~K} / \mathrm{F} 410 \mathrm{~V} / \mathrm{A} 426 \mathrm{~S}}$. 


\section{References:}

(1) Shaner, N. C.; Patterson, G. H.; Davidson, M. W. Advances in Fluorescent Protein Technology. J. Cell Sci. 2007, 120 (Pt 24), 4247-4260. https://doi.org/10.1242/jcs.005801.

(2) Pédelacq, J.-D.; Cabantous, S.; Tran, T.; Terwilliger, T. C.; Waldo, G. S. Engineering and Characterization of a Superfolder Green Fluorescent Protein. Nat. Biotechnol. 2006, 24 (1), 79-88. https://doi.org/10.1038/nbt1172.

(3) Shaner, N. C.; Lambert, G. G.; Chammas, A.; Ni, Y.; Cranfill, P. J.; Baird, M. A.; Sell, B. R.; Allen, J. R.; Day, R. N.; Israelsson, M.; Davidson, M. W.; Wang, J. A Bright Monomeric Green Fluorescent Protein Derived from Branchiostoma Lanceolatum. Nat. Methods 2013, 10 (5), 407-409. https://doi.org/10.1038/nmeth.2413.

(4) Tsien, R. Y. The Green Fluorescent Protein. Annu. Rev. Biochem. 1998, 67 (1), 509-544. https://doi.org/10.1146/annurev.biochem.67.1.509.

(5) Coralli, C.; Cemazar, M.; Kanthou, C.; Tozer, G. M.; Dachs, G. U. Limitations of the Reporter Green Fluorescent Protein under Simulated Tumor Conditions. Cancer Res. 2001, 61 (12), 4784-4790.

(6) Reid, B. G.; Flynn, G. C. Chromophore Formation in Green Fluorescent Protein. Biochemistry 1997, 36 (22), 6786-6791. https://doi.org/10.1021/bi970281w.

(7) Chapman, S.; Faulkner, C.; Kaiserli, E.; Garcia-Mata, C.; Savenkov, E. I.; Roberts, A. G.; Oparka, K. J.; Christie, J. M. The Photoreversible Fluorescent Protein ILOV Outperforms GFP as a Reporter of Plant Virus Infection. Proc. Natl. Acad. Sci. U. S. A. 2008, 105 (50), 2003820043. https://doi.org/10.1073/pnas.0807551105.

(8) Palanisamy, N.; Öztürk, M. A.; Akmeriç, E. B.; Di Ventura, B. C-Terminal EYFP Fusion Impairs Escherichia Coli MinE Function. Open Biol. 10 (5), 200010. https://doi.org/10.1098/rsob.200010.

(9) Roberts, T. M.; Rudolf, F.; Meyer, A.; Pellaux, R.; Whitehead, E.; Panke, S.; Held, M. Identification and Characterisation of a PH-Stable GFP. Sci. Rep. 2016, 6 (1), 28166. https://doi.org/10.1038/srep28166.

(10) Advanced in vivo applications of blue light photoreceptors as alternative fluorescent proteins Photochemical \& Photobiological Sciences (RSC Publishing) https://pubs.rsc.org/en/content/articlelanding/2013/pp/c3pp50040c\#!divAbstract (accessed $2020-11-11)$.

(11) Characterization of flavin-based fluorescent proteins: an emerging class of fluorescent reporters - PubMed https://pubmed.ncbi.nlm.nih.gov/23741385/ (accessed 2020 -11 -11).

(12) Mukherjee, A.; Schroeder, C. M. Flavin-Based Fluorescent Proteins: Emerging Paradigms in Biological Imaging. Curr. Opin. Biotechnol. 2015, 31, 16-23.

https://doi.org/10.1016/j.copbio.2014.07.010. 
(13) Chia, H. E.; Marsh, E. N. G.; Biteen, J. S. Extending Fluorescence Microscopy into Anaerobic Environments. Curr. Opin. Chem. Biol. 2019, 51, 98-104.

https://doi.org/10.1016/j.cbpa.2019.05.008.

(14) Ko, S.; Hwang, B.; Na, J.-H.; Lee, J.; Jung, S. T. Engineered Arabidopsis Blue Light Receptor LOV Domain Variants with Improved Quantum Yield, Brightness, and Thermostability. $J$. Agric. Food Chem. 2019, 67 (43), 12037-12043. https://doi.org/10.1021/acs.jafc.9b05473.

(15) Ko, S.; Jeon, H.; Yoon, S.; Kyung, M.; Yun, H.; Na, J.-H.; Jung, S. T. Discovery of Novel Pseudomonas Putida Flavin-Binding Fluorescent Protein Variants with Significantly Improved Quantum Yield. J. Agric. Food Chem. 2020, 68 (21), 5873-5879.

https://doi.org/10.1021/acs.jafc.0c00121.

(16) Battad, J. M.; Wilmann, P. G.; Olsen, S.; Byres, E.; Smith, S. C.; Dove, S. G.; Turcic, K. N.; Devenish, R. J.; Rossjohn, J.; Prescott, M. A Structural Basis for the PH-Dependent Increase in Fluorescence Efficiency of Chromoproteins. J. Mol. Biol. 2007, 368 (4), 998-1010. https://doi.org/10.1016/j.jmb.2007.02.007.

(17) Henderson, J. N.; Osborn, M. F.; Koon, N.; Gepshtein, R.; Huppert, D.; Remington, S. J. Excited State Proton Transfer in the Red Fluorescent Protein MKeima. J. Am. Chem. Soc. 2009, 131 (37), 13212-13213. https://doi.org/10.1021/ja904665x.

(18) Khrenova, M. G.; Nemukhin, A. V.; Domratcheva, T. Theoretical Characterization of the Flavin-Based Fluorescent Protein ILOV and Its Q489K Mutant. J. Phys. Chem. B 2015, 119 (16), 5176-5183. https://doi.org/10.1021/acs.jpcb.5b01299.

(19) Davari, M. D.; Kopka, B.; Wingen, M.; Bocola, M.; Drepper, T.; Jaeger, K.-E.; Schwaneberg, U.; Krauss, U. Photophysics of the LOV-Based Fluorescent Protein Variant ILOV-Q489K Determined by Simulation and Experiment. J. Phys. Chem. B 2016, 120 (13), 3344-3352. https://doi.org/10.1021/acs.jpcb.6b01512.

(20) Khrenova, M. G.; Meteleshko, Y. I.; Nemukhin, A. V. Mutants of the Flavoprotein ILOV as Prospective Red-Shifted Fluorescent Markers. J. Phys. Chem. B 2017, 121 (43), 10018-10025. https://doi.org/10.1021/acs.jpcb.7b07533.

(21) Oliphant, T. E. Python for Scientific Computing. Comput. Sci. Eng. 2007, 9 (3), 10-20. https:// doi.org/10.1109/MCSE.2007.58.

(22) McKinney, W. Data Structures for Statistical Computing in Python; Austin, Texas, 2010; pp 56-61. https://doi.org/10.25080/Majora-92bf1922-00a.

(23) Hunter, J. D. Matplotlib: A 2D Graphics Environment. Comput. Sci. Eng. 2007, 9 (3), 90-95. https://doi.org/10.1109/MCSE.2007.55.

(24) Goncharov, I. M.; Smolentseva, A.; Semenov, O.; Natarov, I.; Nazarenko, V. V.; Yudenko, A.; Remeeva, A.; Gushchin, I. High-Resolution Structure of a Naturally Red-Shifted LOV Domain. Biochem. Biophys. Res. Commun. 2021, 567, 143-147. https://doi.org/10.1016/j.bbrc.2021.06.046. 
(25) Remeeva, A.; Nazarenko, V. V.; Kovalev, K.; Goncharov, I. M.; Yudenko, A.; Astashkin, R.; Gordeliy, V.; Gushchin, I. Insights into the Mechanisms of Light-Oxygen-Voltage Domain Color Tuning from a Set of High-Resolution X-Ray Structures. Proteins 2021. https://doi.org/10.1002/prot.26078.

(26) Röllen, K.; Granzin, J.; Remeeva, A.; Davari, M. D.; Gensch, T.; Nazarenko, V. V.; Kovalev, K.; Bogorodskiy, A.; Borshchevskiy, V.; Hemmer, S.; Schwaneberg, U.; Gordeliy, V.; Jaeger, K.-E.; Batra-Safferling, R.; Gushchin, I.; Krauss, U. The Molecular Basis of Spectral Tuning in Blue- and Red-Shifted Flavin-Binding Fluorescent Proteins. J. Biol. Chem. 2021, 296, 100662. https://doi.org/10.1016/j.jbc.2021.100662.

(27) Christie, J. M.; Hitomi, K.; Arvai, A. S.; Hartfield, K. A.; Mettlen, M.; Pratt, A. J.; Tainer, J. A.; Getzoff, E. D. Structural Tuning of the Fluorescent Protein ILOV for Improved Photostability. J. Biol. Chem. 2012, 287 (26), 22295-22304. https://doi.org/10.1074/jbc.M111.318881.

(28) Biasini, M.; Bienert, S.; Waterhouse, A.; Arnold, K.; Studer, G.; Schmidt, T.; Kiefer, F.; Cassarino, T. G.; Bertoni, M.; Bordoli, L.; Schwede, T. SWISS-MODEL: Modelling Protein Tertiary and Quaternary Structure Using Evolutionary Information. Nucleic Acids Res. 2014, 42 (W1), W252-W258. https://doi.org/10.1093/nar/gku340.

(29) The PyMOL Molecular Graphics System, Version 1.7.2.1 Schrödinger, L. PyMOL. 